\title{
Práticas educativas como forma de predição de problemas de comportamento e competência social
}

\author{
Educational practices as behavior and social competence \\ problems predicting issues
}

\author{
Caroline Guisantes De SALVO \\ Edwiges Ferreira de Matos SILVARES ${ }^{2}$ \\ Plinio Marco de TONI ${ }^{3}$
}

\begin{abstract}
Resumo
O modelo de Estilo Parental de Gomide é composto por sete práticas educativas avaliadas através de um Inventário de Estilos Parentais: cinco relacionadas ao desenvolvimento de comportamentos anti-sociais (abuso físico, punição inconsistente, disciplina relaxada, monitoria negativa e negligência) e duas favoráveis ao desenvolvimento de comportamentos pró-sociais (monitoria positiva e comportamento moral). O Child Behavior Check List é composto de duas partes, sendo que a primeira se destina à avaliação de competência social e a segunda à de problemas de comportamento. O objetivo deste trabalho foi levantar quais práticas educativas poderiam ser preditoras de comportamentos listados pelo Child Behavior Check List. Participaram deste estudo trinta crianças, com idades entre onze e treze anos, da rede pública de ensino, e um de seus pais. Obteve-se por meio de regressão estatística que monitoria positiva e comportamento moral são variáveis preditoras de comportamentos pró-sociais, e sua falta aliada às práticas negativas são preditoras de distúrbios do comportamento.
\end{abstract}

Palavras-chave: práticas educativas; habilidades sociais; problemas de comportamento.

\begin{abstract}
The Gomide's Parental Style Model (2003) is composed by seven educational practices that can be assessed using the Parental Styles Inventory: five of them are associated to the development of anti-social behaviors (physical abuse, inconsistent punishment, discipline absence, negative monitory and negligence), and two others that are considered propitious to the development of pro-social behaviors (positive monitory and moral behavior). The Child Behavior Check List is composed has two parts: the first assesses social competence, and the second behavior problems. The purpose of this paper was to point out the educational practices associated to the behaviors from the Child Behavior Check List. The sample of this paper was formed by 30 children whose ages were from 11 to 13, all of them studying at public schools. Also, one of each child's parents had participated as a volunteer of this sample. According to the statistic regression method, the data had shown that positive monitoring and moral behavior are pro-social behavior variables, and their absence associated to the negative practices are behavior problemsones.
\end{abstract}

Key words: educational practices, social skills; behavior problems.

1 Mestranda em Psicologia Clínica, Instituto de Psicologia, Universidade de São Paulo. São Paulo, SP, Brasil.

2 Programa de Pós-Graduação em Psicologia Clínica, Universidade de São Paulo. Av. Prof. Mello Moraes, 1721, 05508-900. Correspondência para/Correspondence to: E.F.M. SILVARES.

3 Doutorando em Psicologia da Aprendizagem e do Desenvolvimento Humano. Instituto de Psicologia, Universidade de São Paulo. São Paulo, SP, Brasil. 
A psicologia e as demais áreas de conheci-mento que têm no ser humano seu objeto de estudo têm trabalhado nas últimas décadas para entender os determinantes do surgimento do comportamento pró-social e anti-social. Pesquisas têm apontado a grande importância da relação pai-filho e principalmente para as práticas utilizadas por esses pais na educação de seus filhos (Reppold, Pacheco, Bardagi \& Hutz, 2002; Salvo, 2003; Gomide, 2004). São essas estratégias utilizadas pelos pais com o objetivo de promover a socialização de seus filhos que recebem o nome de práticas educativas (Reppold et al., 2002). Os pais geralmente utilizam uma combinação de estratégias, variando de acordo com a situação.

Para Gomide (2003), as práticas parentais podem desenvolver tanto comportamentos prósociais quanto anti-sociais, dependendo da freqüência e intensidade com que o casal parental as utiliza. E é ao resultado do uso desse conjunto de práticas educativas que a autora denomina estilo parental.

As práticas educativas que podem levar ao desenvolvimento de comportamentos anti-sociais são chamadas de práticas educativas negativas, sendo seis as mais encontradas na literatura: negligência, abuso físico e psicológico, disciplina relaxada, punição inconsistente e monitoria estressante. Quanto às práticas educativas positivas, que colaboram para o adequado desenvolvimento pró-social da criança, destacam-se a monitoria positiva e o comportamento moral (Gomide, 2003).

\section{Práticas educativas positivas}

A monitoria positiva é definida como o conjunto de práticas parentais que envolvem atenção e conhecimento dos pais acerca do local onde o filho se encontra e das atividades que são desenvolvidas pelo mesmo (Dishion \& McMahon, 1998; Stattin \& Kerr, 2000; Gomide, 2001; Gomide, 2003). Os estudos de C Wright e Cullen (2001) e de Cernkovich e Giordano (1987) revelam que práticas parentais como a monitoria positiva, o estabelecimento de regras e o $\delta$ relacionamento estreito entre os membros da família

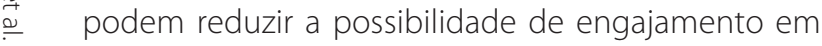
comportamentos de risco por parte do adolescente, ou seja, apontam para a efetividade da monitoria positiva na prevenção de comportamentos anti-
- sociais, demonstrando haver correlação positiva entre apego familiar e comportamentos pró-sociais.

Segundo Gomide (2004), são ainda componentes da monitoria positiva as demonstrações de afeto e carinho dos pais, principalmente as relacionadas aos momentos de maior necessidade da criança, quando, por exemplo, eventos aversivos ocorrem com ela. O apoio e o amor dos pais são a base da monitoria positiva, que, unida ao interesse real pela criança, cria o ambiente propício para a revelação infantil e afasta a necessidade da fiscalização estressante por parte dos pais.

O comportamento moral foi definido por Gomide (2001) como sendo o processo de modelagem de papéis na identificação e nas interações humanas, no que se refere principalmente a normas e valores transmitidos através do modelo parental. Dessa forma, essa prática educativa está ligada aos comportamentos morais transmitidos aos filhos pelos pais, através de seus próprios comportamentos, diálogos etc., referentes a temas como justiça, generosidade, empatia entre outros.

Pesquisas apontam para alguns componentes essenciais para a existência do comportamento moral, sendo eles: sentimento de culpa (Hoffmann, 1975; Loos, Ferreira \& Vasconcelos, 1999), vergonha (Araújo, 1999), empatia (Falcone, 2000), ações honestas (Araújo, 1999; Comte-Sponville, 2000), ações generosas (Araújo,1999; Comte-Sponville, 2000), crenças positivas sobre o trabalho (Mussen, Conger \& Kagan,1974) e ausência de práticas anti-sociais (Patterson, Reid \& Dishion,1992). Todos esses componentes vão sendo desenvolvidos através das relações da criança com o ambiente, onde os pais podem ser os melhores mediadores para favorecer a discriminação e a aquisição de repertório moral. Gomide (2004) salienta a importância de os pais refletirem junto à criança sobre seus comportamentos, ensinando-a a se colocar no lugar dos outros e propiciando situações para que ela repare seus atos no intuito de promover reflexões, desenvolver a empatia e em conseqüência o comportamento moral.

\section{Praticas educativas negativas}

Há muito tempo pesquisadores do comportamento humano têm considerado a negligência como 
fator de risco para o desenvolvimento da criança e do adolescente (Bowlby, 1990; Dodge, Pettit\& Battes, 1994). A negligência foi definida por Gomide (2002) como a prática parental que remete à ausência de supervisão e interesse dos pais em relação à vida de seu filho. Nesses casos, os pais agem como espectadores e não participantes da educação, sendo que essa situação pode se caracterizar por falta de atenção, descaso, omissão e até mesmo falta de amor.

A negligência é considerada um dos principais fatores, senão o principal, a desencadear comportamentos anti-sociais nas crianças, e está associada à história de vida de usuários de álcool e outras drogas e de adolescentes com comportamento infrator (Gomide, 2004).

Em relação à punição inconsistente, segundo Gomide (2001), a falha em se usar eficazmente a punição, independente do humor do agente punidor, éa principal característica dessa prática educativa, ou seja, a punição inconsistente caracteriza-se pela punição dependente do humor dos pais e não em contigüidade ao comportamento da criança; assim, havendo inconstância nas conseqüências do comportamento do filho, este não sabe como agir e aprende mais a discriminar o humor dos pais do que a agir de forma correta. A permanência do comporta-mento indesejado também é uma conseqüência da punição inconsistente, já que ora é punido, ora não (Gomide, 2003).

Em oposição à monitoria positiva, a monitoria negativa se caracteriza pelo excesso de fiscalização da vida dos filhos e pela grande quantidade de instruções repetitivas, que não são seguidas pelos filhos. Essa prática educativa causa ambientes familiares estressados e sem diálogo, uma vez que os filhos podem fazer uso de comportamentos agressivos para proteger dos pais sua privacidade (Gomide, 2003). A monitoria negativa está intimamente ligada também ao controle psicológico. Segundo Pettit, Laird, Dodge, Bates e Criss (2001), o controle psicológico refere-se às tentativas de controle que inibem ou interferem no desenvolvimento de independência e autodirecionamento da criança pelo fato de manter a dependência emocional dos pais. Dessa forma, o uso extensivo do controle psicológico comportamental (indução de culpa, retirada de amor) impediria a emergência da autonomia psicológica, contribuindo ainda para sentimentos de angústia e inadequação.

A disciplina relaxada é caracterizada, por Gomide (2003), pelo não cumprimento de regras estabelecidas. Os pais ameaçam e quando se confrontam com comportamentos opositores e agressivos dos filhos se omitem, não fazendo valer as regras (Gomide, 2004). Na análise do comportamento é aceito que a família, como primeiro contexto socializador da criança, estabelece a base para os padrões comportamentais infantis (Gomide, 2001). Dessa forma, crianças expostas constantemente a práticas educativas de disciplina relaxada estarão em potencial situação de risco para o desenvolvimento de comportamentos delinqüentes, uma vez que os comportamentos de agressividade e de oposição encontram em tais práticas campos propícios para o seu desenvolvimento (Patterson et al., 1992; Simons, Wu, Lin, Gordon \& Conger, 2000).

$\mathrm{O}$ abuso físico infantil tem a literatura mais completa em termos de fatores de risco do que qualquer outra forma de violência familiar pesquisada (Reppold et al., 2002). Segundo Gershoff (2002), a punição corporal diferencia-se do abuso físico uma vez que a primeira se caracteriza pelo uso da força física para corrigir ou controlar um comportamento, mas sem a intenção de machucar. O segundo, no entanto, é o resultado potencial da punição corporal, que resulta das práticas de punição administradas pelos pais e que ferem a criança. São essas práticas que podem desencadear comportamentos delinqüentes, distúrbios psiquiátricos etc. (Haapasalo \& Pokelaa, 1999). Além dessas possíveis conseqüências, Silvares (2004) afirma que crianças que sofrem abuso físico dos pais têm mais probabilidade de sofrerem problemas de saúde, problemas de comportamentos e déficits cognitivos e socioemocionais.

Gomide (2004) salienta que tais práticas podem também gerar crianças apáticas, medrosas, desinteressadas, já que a criança que é espancada freqüentemente pelos pais não discrimina "o certo do errado" em seu comportamento, ficando sem ação no intuito de evitar a surra. Esse tipo de comportamento dos pais atinge o ser da criança e não o mau comportamento. 
Diversas pesquisas (Pinheiro, 2003; Sabbag, 2003; Salvo, 2003; Weber, 2004) procuram demonstrar como as práticas educativas positivas e negativas podem levar ao desenvolvimento de comportamentos pró-sociais e anti-sociais. Observa-se, de forma geral, que os pais, ao utilizarem com maior freqüência de uma ou outra prática, contribuem para que o repertório da criança se desenvolva para um ou outro extremo.

Weber (2004) comparou famílias com alto e baixo índice de comportamento moral no Inventário de Estilos Parentais (IEP) (Gomide, 2003) e encontrou correlação positiva entre o comportamento dos pais e dos filhos. Pinheiro (2003), Sabbag (2003) e Salvo (2003) trabalharam com famílias com alto e baixo índice de estilo parental no IEP (Gomide, 2003), e constataram que práticas negativas estavam positivamente correlacionadas ao comportamento anti-social, bem como ao estresse, ansiedade, agressividade e baixo índice de habilidades sociais, enquanto as práticas parentais positivas estavam correlacionadas positivamente com comportamentos pró-sociais e habilidades sociais. Nesses quatro estudos, observaram-se altas correlações entre as práticas parentais e o comportamento dos filhos.

A fim de ampliar o conhecimento dessas relações, optou-se neste estudo por trabalhar com dois inventários distintos a fim de observar a possível predição de problemas de comportamento e competência social através das práticas educativas levantadas pelo IEP (Gomide, 2003). Assim, o presente artigo tem como objetivo averiguar, através do método de regressão stepwise, quais práticas educativas do IEP (Gomide, 2003) são preditoras de quais problemas de comportamento e competência social descritos pelo Child Behavior Check List (CBCL) (Achenbach, 1991).

\section{Método}

Participaram deste estudo trinta crianças, de ambos os sexos, na faixa etária de onze a treze anos, provenientes de quinze escolas da rede pública de Curitiba, e um de seus pais.

Foram utilizados dois instrumentos para coleta de dados, sendo eles:
1) Inventário de Estilos Parentais (Gomide, 2003), aplicado nos adolescentes. O IEP é composto de 42 questões sobre a forma como os pais os educam. Cada seis questões do IEP dizem respeito a uma das sete práticas abordadas no inventário (duas práticas educativas positivas e cinco negativas). O IEP é calculado somando-se os pontos obtidos nas questões referentes às práticas positivas (monitoria positiva e comportamento moral) que são subtraídos do somatório dos pontos das práticas negativas (punição inconsistente, negligência, disciplina relaxada, monitoria negativa e abuso físico). O índice quando negativo informa a prevalência de práticas educativas negativas e, quando positivo, a presença de práticas positivas no processo educacional.

2) Child Behavior Check List (Achenbach, 1991), respondido pelos pais em relação aos comportamentos dos filhos. O CBCL permite agrupar os resultados em dois âmbitos: 1) competência social e 2) problemas de comportamento. A competência social é agrupada em três escalas: atividade, sociabilidade e escolaridade, sendo que a soma dos escores brutos das três escalas fornece o escore final de competência social. Os problemas de comportamento, por sua vez, são agrupados nas escalas internalização e externalização. A escala de internalização se subdivide: retraimento, queixas somáticas e ansiedade/depressão; já a escala de externalização, em comportamento delinqüente e comportamento agressivo. Há ainda as subescalas de problemas com o contato social, problemas com o pensamento e problemas de atenção. A soma dos escores brutos obtidos nas subescalas comportamentais leva ao distúrbio total de problemas de comportamento. Os resultados ponderados no $\mathrm{CBCL}$ permitem classificar as crianças em: não-clínica, limítrofe e clínica (Bordin, Mari \& Caiero, 1995). Para as escalas sociais, clínicos, escore abaixo de 30, limítrofes, entre 30 e 33 e não-clínico, acima de 33. Para as escalas comportamentais, clínico acima de 70, limítrofe entre 67 e 70, e não-clínico inferior a 67. Para o escore total clínico, acima de 63, limítrofe, entre 60 e 63, e não-clínico, abaixo de 60.

A amostra de crianças foi selecionada por conveniência, de acordo com a possibilidade de os pais e de as crianças preencherem os inventários em um projeto de apoio à criança carente de Curitiba. Assim, as crianças preencheram as duas formas do IEP (para 
mãe e para pai) em uma sala de aula. Os pais foram convidados a responder ao CBCL de acordo com o comportamento dos filhos, em uma das salas do projeto do qual as crianças participam.

A apuração do IEP foi feita de forma manual, utilizando-se da folha de reposta do instrumento com a qual se obteve o índice parental da mãe e do pai, além dos escores para cada prática educativa. A apuração do CBCL deu-se em software específico, com o qual se obteve o escore bruto e padronizado para cada uma das escalas e subescalas do instrumento. A partir dos escores dos dois instrumentos, procedeu-se a uma análise de regressão (método stepwise) através do software Statistical Package for Social Science (SPSS), onde as variáveis independentes selecionadas foram os escores das práticas educativas e o índice de estilo parental final do pai e da mãe, e as variáveis dependentes os escores padronizados das subescalas do CBCL. Os resultados são apresentados a seguir.

\section{Resultados}

Neste estudo, para cada modelo de regressão foram utilizadas as sete variáveis do IEP (maternas e paternas) como possíveis preditoras dos comportamentos medidos pelo CBCL. Foram realizadas análises de regressão múltipla (método stepwise) para verificar quais variáveis do IEP poderiam predizer cada uma das variáveis analisadas pelo CBCL. Por meio da Tabela 1 pode-se observar que para algumas subescalas do $C B C L$, há apenas uma prática educativa preditora, enquanto em outras, mais de uma prática educativa obteve alto índice de coeficiente de determinação, o que mostra que é o conjunto dessas práticas que predizem tal comportamento, como é o caso, por exemplo, da subescala problemas com o pensamento, que tem como variáveis preditoras as práticas negligência e monitoria negativa materna e falta de monitoria positiva materna. Nesses casos, a prática educativa que detém o maior beta padronizado é a variável que mais contribui para a subescala do CBCL, nesse exemplo, a negligência.

De forma geral, os resultados indicaram coeficientes de determinação $\left(R^{2}\right)$ variando de 0,16 a 0,72 ( $1^{\circ}$ quartil: 0,20; mediana: 0,31; $3^{\circ}$ quartil: 0,46), o que significa que as variáveis preditoras (IEP) explicaram de $16 \%$ a $72 \%$ da variância total das variáveis dependentes (CBCL).

Tabela 1. Variáveis do Parental Styles Inventory preditoras do Child Behavior Check List.

\begin{tabular}{|c|c|c|c|c|c|c|c|}
\hline Variável dependente $(\mathrm{CBCL})$ & $\mathrm{R}^{2}$ & $\mathrm{~F}$ & $\mathrm{gl}$ & $p$ & Variáveis preditoras (IEP) & Beta padronizado & $p$ \\
\hline Comportamento agressivo & 0,31 & 10,20 & 1 & $0,004^{* *}$ & Monitoria positiva materna & $-0,55$ & $0,004^{* *}$ \\
\hline Ansiedade/depressão & 0,46 & 9,20 & 2 & $0,001^{* *}$ & $\begin{array}{l}\text { Monitoria negativa materna } \\
\text { Disciplina relaxada materna }\end{array}$ & $\begin{array}{l}0,54 \\
0,40\end{array}$ & $\begin{array}{l}0,002^{* *} \\
0,019^{*}\end{array}$ \\
\hline Problemas com atenção & 0,20 & 5,70 & 1 & $0,026^{*}$ & Monitoria negativa materna & 0,45 & $0,026^{*}$ \\
\hline Problemas com contato social & 0,39 & 14,56 & 1 & $0,001^{* *}$ & Monitoria positiva paterna & $-0,62$ & $0,001^{* *}$ \\
\hline Problemas com pensamento & 0,72 & 18,20 & 3 & $<0,001^{* *}$ & $\begin{array}{l}\text { Negligência materna } \\
\text { Monitoria negativa materna } \\
\text { Monitoria positiva materna }\end{array}$ & $\begin{array}{r}0,72 \\
0,40 \\
-0,27\end{array}$ & $\begin{array}{r}<0,001^{* *} \\
0,003^{* *} \\
0,032^{*}\end{array}$ \\
\hline Internalização & 0,16 & 4,42 & 1 & $0,047^{*}$ & Monitoria negativa materna & 0,40 & $0,047^{*}$ \\
\hline Externalização & 0,43 & 8,20 & 2 & $0,002^{* *}$ & $\begin{array}{l}\text { Monitoria positiva materna } \\
\text { Monitoria negativa materna }\end{array}$ & $\begin{array}{r}-0,56 \\
0,35\end{array}$ & $\begin{array}{l}0,002^{* *} \\
0,040^{*}\end{array}$ \\
\hline Sociabilidade & 0,16 & 4,30 & 1 & $0,040^{*}$ & Monitoria positiva materna & 0,40 & $0,040^{*}$ \\
\hline Atividades & 0,22 & 6,46 & 1 & $0,018^{*}$ & Comportamento moral materno & 0,47 & $0,018^{*}$ \\
\hline Total CS & 0,21 & 5,92 & 1 & $0,023^{*}$ & Comportamento moral materno & 0,45 & $0,023^{*}$ \\
\hline Total PC & 0,53 & 7,80 & 3 & $0,001^{* *}$ & $\begin{array}{l}\text { Monitoria negativa materna } \\
\text { Monitoria positiva paterna } \\
\text { Disciplina relaxada paterna }\end{array}$ & $\begin{array}{r}0,52 \\
-0,45 \\
0,33\end{array}$ & $\begin{array}{l}0,002^{* *} \\
0,007^{* *} \\
0,042^{*}\end{array}$ \\
\hline
\end{tabular}


Para as subescalas do $C B C L$, retraimento e comportamento delinqüente, não foi possível identificar variáveis preditoras.

\section{Discussão}

Neste estudo foi utilizado o método de regressão stepwise, ou seja, de forma aleatória todas as práticas educativas (variáveis independentes) do IEP foram testadas como preditoras das subescalas de comportamento do CBCL (variáveis dependentes). Dessa forma, todas as práticas encontradas como preditoras das subescalas do CBCL deveram-se a relações significativas entre as mesmas. Os resultados apontaram para coeficientes de determinação variando de 0,16 a 0,72, ou seja, as práticas educativas do IEP explicaram de 16\% a 72\% da variância total das escalas e subescalas do CBCL. Enquanto os coeficientes de regressão explicam a variância total, os coeficientes de regressão padronizados indicam a proporção em que as mudanças nas unidades de desvio-padrão da variável preditiva afetam as mudanças no desvio-padrão da variável dependente. Como salientam Floyd, Evans e McGrew (2003), coeficientes de regressão padronizados variando de 0,10 a 0,29 exprimem relações moderadas entre as variáveis, enquanto coeficientes acima de 0,30 representam fortes relações entre as variáveis da equação. Nos modelos de predição formulados na presente pesquisa, os valores negativos dos coeficientes de regressão padronizados variaram de $-0,27$ a $-0,62$, enquanto os valores positivos variaram de 0,33 a 0,72, indicando valores altamente representativos. Em suma, os resultados sugerem que determinadas práticas educativas podem predizer, significativamente, o comportamento da criança.

A literatura (Patterson, Reid \& Dishion, 1992; Matthews, Woodall, Kenyon \& Jacob, 1996; Gomide, 2001; Pettit, Laird, Dodge, Bates, \& Criss, 2001; Reppold et al., 2002; Gomide, 2003; Gomide, 2004) já aponta há muito tempo para essas relações entre as características e práticas adotadas pelos pais e o posterior desenvolvimento de comportamentos pró-sociais e anti-sociais nas crianças, porém são poucos os estudos, que como o presente, comprovam empiricamente

192 essas relações, valendo-se de métodos acurados para comprovação de hipóteses, que ultrapassam os estudos de correlação.

De forma específica, os resultados do presente estudo apontam para monitoria positiva materna, sozinha, como preditora de duas subescalas de comportamento: quando da sua falta, o comportamento agressivo, e quando da sua presença, a sociabilidade.

A monitoria positiva caracteriza-se justamente pelo real interesse e acompanhamento dos pais para com a criança. Através dessa prática, os pais demonstram afeto pelo seu filho, bem como estão disponíveis a contatos físicos (abraços, beijos etc.) e sociais com a criança, de forma que o filho se sente amado e protegido, sem ter seus direitos cerceados ou haver falta de confiança entre pais e filhos, como acontece na monitoria negativa ou supervisão estressante. Assim, observa-se consistência entre a literatura e os resultados obtidos. A falta de monitoria positiva é uma das variáveis responsáveis pelo desenvolvimento do comportamento agressivo, e sua presença é um dos fatores facilitadores para o desenvolvimento da sociabilidade. Segundo Gomide (2003), pais que exercem adequadamente a monitoria positiva tendem a ter elevado repertório de habilidades sociais. Os resultados do presente estudo também apontam que a falta de monitoria positiva paterna leva a déficits na sociabilidade da criança. Para Del Prette e Del Prette (1999), as habilidades sociais estão ligadas ao desenvolvimento saudável de crianças e adolescentes, já que em um ambiente familiar onde se faça uso dessas, provavelmente se desenvolvem crianças com adequado repertório de habilidades sociais, que são imprescindíveis para uma boa sociabilidade.

O comportamento moral materno aparece como prática preditora de atividades e competência social. Essa prática, junto com a monitoria positiva, é a pratica que leva ao desenvolvimento de comportamentos pró-sociais; aqui se encaixam perfeitamente a subescala atividades, que contempla atividades extracurriculares da criança, como grupos (escoteiros, grupos de igreja etc.), bem como a qualidade das atividades desenvolvidas pela criança em relação ao seu grupo e à escala competência social. Segundo Gomide (2003), a prática comportamento moral não se 
refere apenas a comportamentos ligados à justiça e à moral, mas também a comportamento empático, coerência dos pais, possibilidades de acertos e erros por parte da criança, com a adequada reflexão sobre seus atos. Assim, o comportamento moral, ao predizer atividades e competência social, mostra a importância dessa prática como forma de apoio e modelo de repertório para a criança, colaborando para que esta se desenvolva de forma adequada.

A monitoria negativa caracteriza-se pela repetição de instruções, independente delas serem seguidas ou não, pelo tom hostil e de cobrança com que são feitas e pela falta de confiança dos pais em relação aos filhos. Neste estudo, observa-se a monitoria negativa materna como preditora de problemas com a atenção e da escala de internalização. Também, a falta de monitoria positiva e a monitoria negativa materna são as práticas preditoras da subescala externalização. Através desses resultados, pode-se constatar que a monitoria positiva e negativa são variáveis estreitamente relacionadas aos problemas de comporta-mento. Segundo Menegatti (2002), ao longo do desenvolvimento da criança, respostas disfuncionais parecem se desenvolver principalmente na relação da criança com seus cuidadores, e, em seguida, na escola; porém, a influência da relação parental permanece como central devido aos vínculos afetivos. Essa autora inclui algumas operações nas interações pais-filhos que podem levar a criança à depressão e a outros distúrbios internalizantes e externalizantes. Estão entre essas práticas: a falta de reforçamento positivo, o uso de controle aversivo sobre o comportamento da criança, a exigência de altas taxas de desempenho para que a criança receba reforçamento, além da falta de reforçamento diferencial para respostas sociais adequadas (comportamentos socialmente habili-dosos).

Para Lundin (1972), práticas educativas estressoras, como o treinamento perfeccionista, podem gerar crianças muito ansiosas. A falta de reforçamento adequado e a alta taxa de exigência também são características da prática monitoria negativa, uma vez que pais que fazem muito uso dessa prática podem estar sendo regidos mais por regras do que por contingências (Gomide, 2003), não discriminando muitas vezes os reais comportamentos da criança, e podendo contribuir para o desenvolvimento de sintomas de depressão, ansiedade e outros problemas de internalização, externalização, como forma de fuga/ esquiva da criança das situações nas quais o controle parental não tem consistência.

A monitoria negativa materna aliada à disciplina relaxada materna foram preditoras da subescala de ansiedade/depressão do CBCL. Ainda, a monitoria negativa materna aliada à falta de monitoria positiva paterna e a disciplina relaxada paterna foram práticas preditoras do total de problemas de comportamento $(P C)$, variável que surge do somatório de todas as subescalas de problemas de comportamento. A disciplina relaxada é caracterizada pela baixa capacidade dos pais em fazerem valer regras e acordos nas suas interações com os filhos, levando a um ambiente instável na família. Para Malagris e Castro (2000), mães com dificuldades em manter na família um ambiente seguro, devido a sua própria ansiedade (o que pode levar à disciplina relaxada), podem transmitir insegu-rança para seus filhos, favorecendo o desenvolvimento de transtornos de ansiedade, depressão e de outros transtornos internalizantes na infância, devido ao apego inseguro.

Uma das práticas educativas mais comentadas na literatura, devido aos déficits que causam no desenvolvimento infantil, é a negligência (Weber \& Kossobudsky, 1996; Weber, 1998; Gomide, 2003). A negligência materna, a monitoria negativa materna e a falta de monitoria positiva materna foram práticas listadas como preditoras da subescala problemas de pensamento. A literatura aponta para os males da negligência, que por se caracterizar pela ausência de reforçamento, independente do tipo de comportamento que a criança emita, leva muitas vezes ao desenvolvimento do comportamento anti-social, como apontam diversas pesquisas com crianças institucionalizadas ou abandonadas na própria família (Weber \& Kossobudsky, 1996; Weber, 1998). Por outro lado, a negligência aliada à monitoria negativa, em um primeiro momento, pode parecer antagônica, já que a segunda se caracteriza por uma fiscalização constante dos pais, que vai justamente em caminho oposto à negligência. Porém, vale ressaltar que quando os filhos são extremamente fiscalizados, não se sentem 
amados, mas pelo contrário, atribuem isso à falta de amor e de respeito dos pais (Gomide, 2004). Olhando-se dessa forma, a negligência apontada se deve justamente à falta de monitoria positiva apontada pela regressão estatística, já que os pais não demonstram real interesse pela criança, o que pode caracterizar a negligência e a falta de monitoria positiva ao mesmo tempo.

\section{Considerações Finais}

Este estudo procurou contribuir com o que é mais caro na psicologia: a prevenção. Identificando-se as práticas parentais preditoras de quais comportamentos infantis, abre-se a possibilidade de ampliação de projetos e práticas preventivas, de caráter focal, possíveis de serem aplicadas em escolas, comunidades, clínicas etc. O adequado desenvolvimento infantil é o somatório de diversos fatores, porém, os pais estão entre os mais importantes. Este estudo buscou apontar uma direção nesse caminho, porém, ainda muito pode ser feito para contribuir para o entendimento do relacionamento entre pais e filhos.

\section{Referências}

Achenbach, T.M. (1991). Manual for the child behaviorchecklist/ 4-18 and 1991 profile(VT 1-288). Burlington: University of Vermont.

Araújo, U.F. (1999). Conto de escola: a vergonha como um regulador moral. São Paulo: Editora Moderna.

Bowlby, J. (1990). Apego: a natureza do vínculo. São Paulo: Martins Fontes.

Bordin, I.A.S., Mari, J.J., \& Caiero, M.F. (1995). Validação da versão brasileira do Child Behavior Checklist $(\mathrm{CBCL})$ Inventário de comportamentos da Infância e adolescência: dados preliminares. Revista ABP APPAL, 17 (2), 55-66.

Cernkovich, S.A., \& Giordano, P.C. (1987). Family relationships and delinquency. Criminology, 25 (2), 295-321.

Comte-Sponville, A. (2000). Pequeno tratado das grandes virtudes. São Paulo: Martins Fontes.

Del Prette, A., \& Del Prette, Z.A.P. (1999). Psicologia das habilidades sociais: terapia e educação. Petrópolis: Vozes.

Dishion, T.J., \& McMahon, R.J. (1998). Parental monitoring and the prevention of child and adolescent problem behavior: a conceptual and empirical formulation. Clinical Child and Family Psychology Review, 1 (1), 61-75.
Dodge, K.A., Pettit, G.S., \& Battes, J.E. (1994). Socialization mediators of the relation between socioeconomic status and child conduct problems. Child Development, 65 (2), 649-665.

Falcone, E. (2000). Habilidades sociais: para além da assertividade. In R.C. Wielenska (Org.). Sobre comportamento e cognição (v.6). Santo André; Esetec.

Floyd, R.G., Evans, J.J., \& McGrew, K.S. (2003). Relations between measures of Cattell-Horn-Carroll (CHC) cognitive abilities and mathematics achievement across the school-age years. Psychology in the Schools, 40 (2), 155-171.

Gershoff, E.T. (2002). Corporal Punishment by parents and associated child behaviors and experiences: a meta-analytic and theoretical review. Psychological Bulletin, 128 (4), 539-579.

Gomide, P.I.C. (2001). Efeito das práticas educativas no desenvolvimento do comportamento anti-social. In M.L. Marinho \& V.E. Caballo (Orgs.). Psicologia clínica e da saúde. Londrina: UEL.

Gomide, P.I.C. (2002). Crianças e adolescentes em frente à TV: o que e quanto assistem televisão. Psicologia Argumento, 30 (19), 17-28.

Gomide, P.I.C. (2003). Estilos Parentais e comportamento anti-social. In A. Del Prette \& Z. Del Prette (Orgs.). Habilidades sociais, desenvolvimento e aprendizagem: questões conceituais, avaliação e intervenção (21-60). Campinas: Alínea.

Gomide, P.I.C. (2004). Pais presentes, pais ausentes. Petrópolis: Vozes.

Haapasalo, J., \& Pokelaa, E. (1999) Child-rearing and abuse antecedents of criminality. Journal of Aggression and Violent Behavior, 4 (1), 107-127.

Hoffman, M.L. (1975). Moral internalization, parental power and the nature of Parent-Child Interaction. Developmental Psychology, 11 (2), 228-239.

Loos, H., Ferreira, S.P.A., \& Vasconcelos, F.C. (1999). Julgamento moral: estudo comparativo entre crianças institucionalizadas e crianças de comunidade de baixa renda com relação à emergência do sentimento de culpa. Psicologia: Reflexão e Crítica, 12 (1), 65-100.

Lundin, R.W. (1972). Personalidade: uma análise do comportamento. São Paulo: Edusp.

Malagris, L.E.N., \& Castro, M.A. (2000). Distúrbios emocionais e elevações de stress em crianças. In M.E.N Lipp (Org.). Crianças estressadas: causas, sintomas e soluções (pp.65-100). Campinas: Papirus.

Matthews, K.A., Woodall, K.L., Kenyon, K., \& Jacob, T. (1996). Negative family environment as a predictor of boys's future status on measures of hostile attitudes, interview behavior, and anger expression. Health Psychology, 15 (1), 30-37.

Menegatti, C.L. (2002). Estilos parentais e depressão infantil. Dissertação de mestrado não - publicada, Universidade Federal do Paraná, Curitiba.

Mussen, P.H., Conger, J.J., \& Kagan, J. (1974). Desenvolvimento e personalidade da criança. São Paulo: Harbra. 
Patterson, G.R., Reid, J.B., \& Dishion, T.J. (1992). Antisocial boys. New York: Castalia Publishing Company.

Pettit, G., Laird, R.D., Dodge, K.A., Bates, J., \& Criss, M. (2001). Antecedents and behavior-problem outcomes of parental monitoring and psychological control in early adolescence. Child Development, 72 (2), 583-598.

Pinheiro, D.P.N. (2003). Estilo parental: uma análise qualitativa. Dissertação de mestrado, Universidade Federal do Paraná, Curitiba.

Reppold, C.T., Pacheco, J., Bardagi, M., \& Hutz, C.S. (2002). Prevenção de problemas de comportamento e desenvolvimento de competências psicossociais em crianças e adolescentes: uma análise das práticas educativas e dos estilos parentais. In S.C. Hutz (Org.) Situações de risco e vulnerabilidade na infância e na adolescência: aspectos teóricos eestratégia de intervenção (pp. 7-52). São Paulo: Casa do Psicólogo.

Sabbag, G.M. (2003). Validação Externa do Inventário de Estilos Parentais: um estudo de caso com duas famílias de risco. Monografia de conclusão do curso de psicologia, Universidade Federal do Paraná, Curitiba.

Salvo, C.G. (2003). Validação Externa do Inventário de Estilos Parentais: estudo de caso com famílias pró-sociais. Monografia de conclusão do curso de psicologia, não-publicada, Universidade Federal do Paraná, Curitiba.
Simons, R.L., Wu, C., Lin, K., Gordon, L., \& Conger, R. (2000). A cross-cultural examination of the link between corporal punishment and adolescent antisocial behavior. Criminology, 38 (1), 47-80.

Silvares, E.F.M.S. (2004). Avaliação diagnóstica do abuso físico na infância: implicações clínicas e de pesquisa. In M.Z.S. Brandão (Org.). Sobre comportamento e cognição, 14 (pp.19-37). Santo André: ESETec Editores Associados.

Stattin, H., \& Kerr, M. (2000). Parental monitoring: a reinterpretation. Child Development, 71 (4), 1072-1085.

Weber, L. (2004). Efeito do comportamento moral dos pais sobre o comportamento moral dos filhos adolescentes. Dissertação de mestrado, Universidade Federal do Paraná, Curitiba.

Weber, L.N.D. (1998). Laços de ternura: pesquisa e histórias de adoção. Curitiba: Santa Mônica.

Weber, L.N.D., \& Kossobudsky, L.H.M. (1996). Filhos da solidão. Curitiba: Governo do Estado do Paraná.

Wright, J.P., \& Cullen, F.T. (2001). Parental efficacy and delinquent behavior: do control and support matter? Criminology, 39 (3), 677-705.

Recebido para publicação em 5 de janeiro e aceito em 17 de março de 2005 
